\title{
Current status of intensified neo-adjuvant systemic therapy in locally advanced rectal cancer
}

\section{Benedikt Engels, Thierry Gevaert, Alexandra Sermeus and Mark De Ridder *}

Department of Radiotherapy, UZ Brussel, Vrije Universiteit Brussel, Brussels, Belgium

Edited by:

Tim Greten, National Cancer Institute, USA

\section{Reviewed by:}

Arndt Vogel, Medical School

Hannover, Germany

Mirko Omejc, University Medical

Centre Ljubljana, Slovenia

Florian Lordick, Klinikum

Braunschweig, Germany

*Correspondence:

Mark De Ridder, Department of Radiotherapy, UZ Brussel, Vrije

Universiteit Brussel, Laarbeeklaan 101, 1090 Brussel.

e-mail: mark.deridder@uzbrussel.be
The addition of 5-fluorouracil (5-FU) or its prodrug capecitabine to radiotherapy (RT) is a standard approach in the neo-adjuvant treatment of patients with rectal tumors extending beyond the muscularis propria (stage II) and/or with clinical evidence of regional lymph node metastases (stage III). According to European randomized trials, the combined treatment modality resulted in favorable local control rates as compared with radiotherapy (RT) alone, but no improvement was found regarding the occurrence of distant metastases or overall survival. In an effort to further enhance the response rates and to decrease the high incidence of distant metastases in locally advanced rectal cancer patients, the addition of other chemotherapeutical drugs and biologic agents as radiation sensitizers to neo-adjuvant 5-FU based chemoradiotherapy (CRT) has been recently investigated. The role of those agents is however questionable as first results from phase III data do not show improvement on pathologic complete remission and circumferential resection margin negative resection rates as compared to 5-FU based CRT, nevertheless an increased toxicity.

Keywords: rectal cancer, neo-adjuvant radiotherapy, chemoradiotherapy

\section{INTRODUCTION}

Over the past decades, considerable advances have been made in the use of long-course CRT in rectal cancer. Based on a number of pivotal studies in the US which demonstrated positive effects on local control (LC) and overall survival (OS) with the addition of RT and chemotherapy following surgical resection in locally advanced rectal cancer, most clinicians initially favored the use of adjuvant 5-FU based CRT (Krook et al., 1991; Wolmark et al., 2000). Theoretically, RT should be delivered neo-adjuvantly rather than adjuvantly since poor cell oxygenation in the healing tumor bed potentially decreases radiosensitivity. Secondary, as the small bowel is not trapped in the pelvic bay by postoperative adhesions, fewer enteric complications are expected with neo-adjuvant RT, where moving loops of small bowel are located on different positions from day to day. Lastly, neo-adjuvant downstaging may positively influence the surgical margin status and sphincter preservation rate. Strong evidence of the superiority of neo-adjuvant to adjuvant CRT in stage II/III rectal cancer patients undergoing total mesorectal excision (TME) was provided by the 5-year results of the randomized German Rectal Cancer Study (CAO/ARO/AIO 94), with a significant lower rate of pelvic recurrence in favor of neo-adjuvant CRT as compared to adjuvant CRT (6 versus 13\%). Moreover, the data also showed significant lower rates of toxicity with the use of neo-adjuvant CRT (Sauer et al., 2004). The addition of 5-FU to long-course neoadjuvant RT is considered standard of care in locally advanced rectal cancer by the evidence of two important phase III trials, EORTC 22921 and FFCD 9203, which demonstrated the advantages of neo-adjuvant 5-FU based CRT over RT alone with respect to pathologic complete remission (pCR) and LC, but not with respect to the rate of sphincter-sparing surgery, occurrence of distant metastases, or OS (Bosset et al., 2006; Gérard et al., 2006).
Those findings led to a shift in the European and US policy of adjuvant CRT to neo-adjuvant 5-FU CRT, which is now standard of care in stage II/III rectal cancer. Recently, a non-inferiority phase III trial of the Mannheimer Arbeitsgruppe für Gastrointestinale Tumoren (MARGIT) which compared capecitabine to 5-FU in (neo-)adjuvant CRT of locally advanced rectal cancer provided a growing body of evidence for using capecitabine (Hofheinz et al., 2011). Besides a non-inferiority to 5-FU regarding 5-year OS (76 versus $67 \%$ with 5 -FU, $p<0.05$ ), patients receiving capecitabine experienced equal LC. In concordance with the toxicity profile of capecitabine in the adjuvant treatment of colorectal cancer (CRC), less leukopenia but more hand-foot skin reactions and proctitis were recorded compared to 5-FU (Hofheinz et al., 2011). In view of its advantageous administration profile (no need for catheter placement and infusion pump), those encouraging findings may impact the current standard of administrating 5-FU intravenously during neo-adjuvant RT. Despite those improvements, more than one third of the patients undergoing curative treatment of rectal cancer have distant recurrence of this disease. In an effort to further improve the pCR rate, which is considered as a surrogate end point for LC and OS, and to decrease the high incidence of distant recurrences, several phase II and III trials investigated in addition to neo-adjuvant 5-FU based CRT the use of induction chemotherapy and multi-agent systemic therapy by means of other chemotherapeutical drugs (oxaliplatin) or biologic agents such as cetuximab and bevacizumab, which are epidermal growth factor receptor (EGFR) and vascular endothelial growth factor (VEGF) directed monoclonal antibodies, respectively.

\section{INDUCTION CHEMOTHERAPY}

An attempt to enhance the outcome after neo-adjuvant CRT and TME includes the concept of induction chemotherapy, which has 
been reported so far only in phase II setting (Chua et al., 2010; Fernandez-Martos et al., 2010). A randomized phase II Spanish trial observed no differences in $\mathrm{pCR}$ and $\mathrm{R} 0$ resection rate between patients receiving neo-adjuvant CRT with capecitabine and oxaliplatin (CAPOX) and those receiving induction CAPOX followed by CRT with CAPOX (Fernandez-Martos et al., 2010). After a median follow-up of 39 months, similar 3-year disease-free survival (DFS) and OS rates were recorded in the two treatment arms (Fernandez-Martos et al., 2011). The EXPERT trial, carrying out four cycles of neo-adjuvant CAPOX followed by concomitant capecitabine based CRT prior to TME in magnetic resonance imaging (MRI) defined high-risk rectal cancer patients, reported a promising 20\% pCR and 4\% R1 rate, with acceptable safety (Chua et al., 2010). One should however notice the 9 clinically significant cardiotoxic events in 8 out of 77 enrolled patients, of which 4 patients died, before protocol amendment of this trial in January 2004 to exclude patients with recent cardiac morbidity (Chau et al., 2006). Although the impressive number of early fatal events in this trial is not observed in other studies evaluating the safety of induction chemotherapy, acute toxicity is considerable with reported rates of grade $\geq 3$ acute diarrhea and lymphocytopenia of 20 and $43 \%$, respectively, according to a phase II study where patients received one cycle of CAPOX followed by CAPOX based CRT and surgery (Koeberle et al., 2008). Based on those phase II data, induction chemotherapy does not seem to be safe and should therefore not be recommended outside clinical trials.

\section{ADDITION OF OXALIPLATIN TO NEO-ADJUVANT 5-FU BASED CRT}

Four randomized phase III studies, the Studio Terapia Adiuvante Retto (STAR)-01 trial, Prodige 2-ACCORD-12/405 trial, the National Surgical Adjuvant Breast and Bowel Project (NSABP) R04 trial and the German CAO/ARO/AIO-04 trial evaluated the tolerability and efficacy of the addition of oxaliplatin to 5-FU based CRT as neo-adjuvant treatment of locally advanced rectal cancer (Gérard et al., 2010; Aschele et al., 2011; Roedel et al., 2011; Roh et al., 2011). No improvement in the pCR rate was noted as compared to 5-FU based CRT alone in the STAR-01 trial, with reported rates of $16 \%$ in both arms (Aschele et al., 2011). Furthermore, no differences in $\mathrm{R} 0$ resection rates circumferential resection margin $(\mathrm{CRM} ;>1 \mathrm{~mm})$ were observed with the addition of oxaliplatin (93 versus 96\%; Aschele et al., 2011). Longer follow-up is needed in this study to assess the impact on the primary endpoint, OS. In similarity, the Prodige 2-ACCORD-12/405 trial displayed no differences in pCR (19 versus $14 \%, p=0.09$ ), the primary endpoint, or CRM involvement ( 7.7 versus $12.7 \%$, $p=0.17)$ with the addition of oxaliplatin to capecitabine based CRT (Gérard et al., 2010). Those findings were confirmed by first results from the NSABP R-04 trial, randomizing 1680 rectal cancer patients between four treatment groups: neo-adjuvant continuous infusion 5-FU CRT with or without oxaliplatin or neo-adjuvant capecitabine CRT with or without oxaliplatin, showing no differences in downstaging, sphincter preservation rate or pCR (Roh et al., 2011). Primary endpoints of this study, DFS and OS, are awaiting. The cumulative incidence of distant recurrence of $36 \%$ in the neo-adjuvant arm of the German Rectal Cancer Study (Sauer et al., 2004) led to a comparison of this regimen to an arm that incorporates oxaliplatin during both neo-adjuvant 5-FU CRT and 5-FU adjuvant chemotherapy in the German CAO/ARO/AIOO4 randomized phase III trial, which was powered to detect a $7 \%$ difference in 3-year DFS as primary endpoint (Roedel et al., 2011). First results of this trial showed no significant differences in grade $\geq 3$ acute toxicity ( $22 \%$ for 5 -FU versus $23 \%$ for 5 FU/oxaliplatin arm), rates of sphincter preserving surgery, and R0 resection, whereas a slight but significant improvement was found in terms of pCR with the addition of oxaliplatin (17 versus $13 \%$, $p=0.045$; Roedel et al., 2011). It should be noticed that their regimen, unlike the other trials, included a chemotherapy gap during the third week of RT which may explain the similar toxicity rates and improved compliance rates (Roedel et al., 2011). Additional follow-up is however needed to report on the primary endpoint. Recently, Gérard et al. (2012) presented the clinical outcomes after a median follow-up time of 36 months of the 598 patients included in the Prodige 2-ACCORD-12/405 trial, and they observed no differences in LC, DFS, or OS between the group of patients who received neo-adjuvant CAPOX based CRT as compared to those who underwent neo-adjuvant capecitabine CRT. Not surprisingly with intensified multi-agent chemotherapy, side effects may be enhanced significantly. First results from the NSABP R-04 trial displayed a more than twofold excessive risk of grade $\geq 3$ diarrhea with the addition of oxaliplatin to 5-FU based CRT (Roh et al., 2011). The STAR-01 and Prodige 2-ACCORD-12/405 trial reported a significant increase in grade $\geq 3$ acute toxicity, with reported rates of $25 \%$ in patients receiving oxaliplatin compared to $8-11 \%$ for the groups receiving $5-F U$ based CRT alone (Gérard et al., 2010; Aschele et al., 2011). Given the activity of oxaliplatin in combination with 5-FU based chemotherapy in the adjuvant and metastatic setting of colon cancer and the well established radiosensitizing effects of another platinum-based drug, cisplatin, in the treatment of lung cancer and cervical cancer, its clinical additive effect to 5-FU based CRT in the neo-adjuvant setting of rectal cancer is disappointing. Regarding its active launch into clinical combined modality trials, with the first phase I study of oxaliplatin in neo-adjuvant CRT of rectal cancer already 11 years ago (Freyer et al., 2001), solid biological data with respect to the radiosensitizing activity of oxaliplatin are quite limited and not conclusive. Only in abstract form, oxaliplatin was shown to sensitize CRC cell cultures to radiation in vitro (Blackstock et al., 2000, 2002). In combination with 5-FU in vitro, exposure of human CRC cell line HT29 to oxaliplatin indicated synergistic effects to radiation, whereas in vivo it failed to improve the growth inhibitory response induced by fractionated radiation doses of $2 \mathrm{~Gy}$ plus capecitabine in HT29 colorectal tumor xenografts (Folkvord et al., 2008). On the basis of the scarcity of this preclinical evidence, further experimental in vivo studies are warranted to determine the radiosensitizing properties of oxaliplatin in neo-adjuvant CRT of rectal cancer and to provide a strong preclinical knowledge which is critical before moving on to further phase III trials. Moreover, the primary goal in those phase III trials is of crucial concern in the era of 5-FU based CRT, where local recurrences after TME are already reported to occur in less than $5 \%$ of the patients and where surrogate end points such as pCR are debatable. Given that one third of the patients with resected rectal cancer develop metastatic disease despite the very high LC after neo-adjuvant CRT and TME, 
the focus should be on the eradication of subclinical metastatic disease. However, with current clinical end points such as DFS and OS, the large number of patients to be enrolled and long time period before analysis within phase III studies do not allow a quick answer on the systemic efficacy of a new medical treatment in the neo-adjuvant setting of rectal cancer. In conclusion, the addition of oxaliplatin to 5-FU based CRT cannot be considered a standard approach in the neo-adjuvant management of locally advanced rectal cancer, but the long-term follow-up data of those phase III trials should be awaited.

\section{CETUXIMAB}

In a randomized phase II study, the EXPERT-C trial addressed the addition of weekly cetuximab to the concept of induction CAPOX and concurrent capecitabine CRT, as used in the EXPERT trial (Chua et al., 2010), in high-risk rectal cancer patients (Dewdney et al., 2011). No differences were found in pCR, progression-free survival (PFS) and OS for the all treated population (Dewdney et al., 2011). In subgroup analysis, KRAS and BRAF wild type patients displayed significantly improved 3-year OS with the addition of cetuximab once per week throughout treatment compared to those who received induction chemotherapy and CRT alone (96 versus $81 \%, p=0.04$; Dewdney et al., 2011). Interestingly, there was no significant difference in pCR (7 versus $11 \%$ ) in the wild type patients receiving cetuximab compared to those not receiving cetuximab (Dewdney et al., 2011). The low pCR rate may in part be explained by the eight patients displaying a pCR but with insufficient tissue for KRAS and BRAF analysis, of which six patients received cetuximab. Grade $3+$ skin rash was, as expected, increased with the use of cetuximab as well as the rates of diarrhea in patients receiving cetuximab, the latter only during CRT (Dewdney et al., 2011). In a pooled analysis of 10 phase I/II trials incorporating cetuximab in neo-adjuvant CRT of rectal cancer, an overall pCR rate and R1 resection rate of 9 and 7\% were reported (Glynne-Jones et al., 2010). Based on those first results, adding cetuximab to neo-adjuvant CRT does not seem to improve pCR, with respect to an overall pCR rate of $13 \%$ seen after 5 -FU based CRT (Glynne-Jones et al., 2010). Possible reasons for the disappointing results on the early endpoint of pCR include a less crucial role for repopulation in adenocarcinoma of the rectum in the presence of continuous exposure to 5-FU and RT (Brierley et al., 1996; Glynne-Jones et al., 2010). In addition, cetuximab could potentially abolish the additive effects of 5 -FU by blockade of $S$ phase entry, as cells which fail to progress through $S$ phase do not accumulate additive effects from the combination of 5FU and radiation (Lawrence et al., 1996, 2003; Huang and Harari, 2000). The optimum sequence of cetuximab in addition to 5 -FU

\section{REFERENCES}

Aschele, C., Cionini, L., Lonardi, S., Pinto, C., Cordio, S., Rosati, G., Artale, S., Tagliagambe, A., Ambrosini, G., Rosetti, P., Bonetti, A., Negru, M. E., Tronconi, M. C., Luppi, G., Silvano, G., Corsi, D. C., Bochicchio, A. M., Chiaulon, G., Gallo, M., and Boni, L. (2011).
Primary tumor response to preoperative chemoradiation with or without oxaliplatin in locally advanced rectal cancer: pathologic results of the STAR-01 randomized phase III trial. J. Clin. Oncol. 29, 2773-2780.

Blackstock, A. W., Tepper, J., and Hess, S. (2000). Oxaliplatin: in vitro and in vivo evidence of its radiation

CRT remains unclear and should be explored before moving on to phase III trials. Furthermore, biomarkers such as KRAS and $B R A F$ mutational status, which are predictive for lack of response to anti-EGFR therapy in metastatic CRC, have to be integrated in those trials, finally to help us in selecting out appropriate patients which may benefit from neo-adjuvant CRT in combination with cetuximab.

\section{BEVACIZUMAB}

In a Phase I/II trial in rectal cancer patients receiving bevacizumab and CRT, Willett et al. (2004) provided direct evidence of the antivascular effect of anti-VEGF treatment by functional, cellular, and molecular investigations. Briefly, bevacizumab decreases the tumor vascular density, tumor perfusion, tumor interstitial fluid pressure, and the number of viable circulating endothelial and progenitor cells, which results into a significant increase in apoptosis of cancer cells (Willett et al., 2004). Several phase I/II trials reported on the feasibility of adding bevacizumab to 5-FU based CRT in the neo-adjuvant setting of locally advanced rectal cancer, and provided encouraging pCR rates with moderate toxicity (Willett et al., 2009; Crane et al., 2010). The reported incidence of postoperative wound complications in up to $36 \%$ of the patients is however concerning and consistent with other reports utilizing bevacizumab with CRT before a major surgical procedure (Dipetrillo et al., 2012). Recently, a systematic review identified between 2000 and 201115 trials which incorporated bevacizumab in neo-adjuvant CRT and calculated a pooled pCR rate of $21 \%$, which is not superior than those reported with 5-FU based CRT (Shaalan Beg et al., 2012). Taking into account the lack of phase III data in the neo-adjuvant setting of rectal cancer, the similar response rates as compared to 5-FU based CRT alone and the considerable treatment-related toxicities, bevacizumab as a radiation sensitizer in combination with 5-FU based CRT does not seem to provide additional benefits in the neo-adjuvant treatment of locally advanced rectal cancer.

\section{CONCLUSION}

In conclusion, 5-FU based CRT still remains the standard of care in the neo-adjuvant treatment of locally advanced rectal cancer. Intensifying this regimen by the concept of induction chemotherapy, concurrent multi-agent chemotherapy or the addition of biologic agents does not seem to improve treatment efficacy in terms of pCR, sphincter preservation, or CRM negative resection rate, and is therefore not recommended outside clinical trials in locally advanced rectal cancer. The impact of those strategies on the occurrence of distant metastases and OS remains unclear until additional follow-up is achieved.

sensitizing activity. Int. J. Radiat. Oncol. Biol. Phys. 46, 724.

Blackstock, A. W., Tepper, J. E., and Case, L. D. (2002). In vitro and in vivo radiosensitization of colon cancer cells by cis-( (1R2R)-1,2cyclohexanediamine-N,N'(oxalo (2-)-O,O'(platinum (oxaliplatin). Int. J. Mol. Med. 10, S68.
Bosset, J. F., Collette, L., Calais, G., Mineur, L., Maingon, P., RadosevicJelic, L., Daban, A., Bardet, E., Beny, A., Ollier, J. C., and EORTC Radiotherapy Group Trial 22921. (2006). Chemotherapy with preoperative radiotherapy in rectal cancer. N. Engl. J. Med. 355, 1114-1123. 
Brierley, J. D., Keane, T. J., and Cummings, B. (1996). The absence of an adverse effect of prolongation of radiation treatment of primary rectal adenocarcinoma. Clin. Oncol. 8, 97-101.

Chau, I., Brown, G., Cunningham, D., Tait, D., Wotherspoon, A., Norman, A. R., Tebbutt, N., Hill, M., Ross, P. J., Massey, A., and Oates, J. (2006). Neoadjuvant capecitabine and oxaliplatin followed by synchronous chemoradiation and total mesorectal excision in magnetic resonance imaging-defined poor-risk rectal cancer. J. Clin. Oncol. 24, 668-674.

Chua, Y. J., Barbachano, Y., Cunningham, D., Oates, J. R., Brown, G., Wotherspoon, A., Tait, D., Massey, A., Tebbutt, N. C., and Chau, I. (2010). Neoadjuvant capecitabine and oxaliplatin before chemoradiotherapy and total mesorectal excision in MRI-defined poor-risk rectal cancer: a phase 2 trial. Lancet Oncol. 11, 241-248.

Crane, C. H., Eng, C., Feig, B. W., Das, P., Skibber, J. M., Chang, G. J., Wolff, R. A., Krishnan, S., Hamilton, S., Janjan, N. A., Maru, D. M., Ellis, L. M., and RodriguezBigas, M. A. (2010). Phase II trial of neoadjuvant bevacizumab (BEV), Capecitabine (CAP), and radiotherapy (XRT) for locally advanced rectal cancer. Int. J. Radiat. Oncol. Biol. Phys. 76, 824-830.

Dewdney, A., Capdevilla, J., Glimelius, B., Cervantes, A., Tait, D. M., Brown, G., Wotherspoon, A., Gonzalez de Castro, D., Chua, Y. J., Wong, R., Barbachano, Y., Oates, J. R., Chau, I., and Cunningham, D. (2011). EXPERT-C: a randomized, phase II European multicenter trial of neoadjuvant capecitabine plux oxaliplatin chemotherapy (CAPOX) and chemoradiation (CRT) with or without cetuximab followed by total mesorectal excision (TME) in patients with MRI-defined, highrisk rectal cancer. J. Clin. Oncol. 29, abstr. 3513.

Dipetrillo, T., Pricolo, V., LagaresGarcia, J., Vrees, M., Klipfel, A., Cataldo, T., Sikov, W., McNulty, B., Shipley, J., Anderson, E., Khurshid, H., Oconnor, B., Oldenburg, N. B., Radie-Keane, K., Husain, S., and Safran, H. (2012). Neoadjuvant bevacizumab, oxaliplatin, 5-fluorouracil and radiation for rectal cancer. Int. J. Radiat. Oncol. Biol. Phys. 82, 124-129.

Fernandez-Martos, C., Pericay, C., Aparicio, J., Salud, A., Safont, M.,
Massuti, B., Vera, R., Escudero, P., Maurel, J., Marcuello, E., Mengual, J. L., Saigi, E., Estevan, R., Mira, M., Polo, S., Hernandez, A., Gallen, M., Arias, F., Serra, J., and Alonso, V. (2010). Phase II, randomized study of concomitant chemoradiotherapy followed by surgery and adjuvant capecitabine plux oxaliplatin (CAPOX) compared with induction CAPOX followed by concomitant chemoradiotherapy and surgery in magnetic resonance imaging-defined, locally advanced rectal cancer: Grupo Cancer de Recto 3 study. J. Clin. Oncol. 28, 859-865.

Fernandez-Martos, C., Pericay, C., Salud, A., Massuti, B., Alonso, V., Safont, M. J., Vera, M., and Aparicio, M. J. (2011). Three-year outcomes of GCR-3: a phase II randomized trial comparing conventional preoperative chemoradiation (CRT) followed by surgery and postoperative adjuvant chemotherapy (CT) with induction CT followed by CRT and surgery in locally advanced rectal cancer. J. Clin. Oncol. 29, abstr. 3552.

Folkvord, S., Flatmark, K., Seierstad, T., Roe, K., Rasmussen, H., and Ree, A. H. (2008). Inhibitory effects of oxaliplatin in experimental radiation treatment of colorectal carcinoma: does oxaliplatin improve 5-fluorouracil-dependent radiosensitivity? Radiother. Oncol. 86, 428-434.

Freyer, G., Bossard, N., Romestaing, P., Mornex, F., Chapet, O., TrilletLenoir, V., and Gérard, J. P. (2001). Addition of oxaliplatin to continuous fluorouracil, I-folinic acid, and concomitant radiotherapy in rectal cancer: the Lyon R 97-03 phase I trial. J. Clin. Oncol. 19, 2433-2438.

Gérard, J. P., Azria, D., GourgouBourgade, S., Conroy, T., and Bedenne, L. (2012). Clinical results at 3 years of the ACCORD 12 randomized trial in rectal cancer. J. Clin. Oncol. 30, abstr. 389.

Gérard, J. P., Azria, D., GourgouBourgade, S., Martel-Laffay, I., Hennequin, C., Etienne, P. L., Vendrely, V., Francois, E., de La Roche, G., Bouché, O., Mirabel, X., Denis, B., Mineur, L., Berdah, J. F., Mahé, M. A., Bécouarn, Y., Dupuis, O., Lledo, G., Montoto-Grillot, C., and Conroy, T. (2010). Comparison of two neoadjuvant chemoradiotherapy regimens for locally advanced rectal cancer: results of the phase III trial ACCORD 12/405Prodige 2. J. Clin. Oncol. 28, 1638-1644.
Gérard, J. P., Conroy, T., Bonnetain F., Bouché, O., Chapet, O., ClosonDejardin, M. T., Untereiner, M., Leduc, B., Francois, E., Maurel, J., Seitz, J. F., Buecher, B. Mackiewicz, R., Ducreux, M., and Bedenne, L. (2006). Preoperative radiotherapy with or without concurrent fluorouracil and leucovorin in T3-4 rectal cancers: results of FFCD 9203. J. Clin. Oncol. 24, 4620-4625.

Glynne-Jones, R., Mawdsley, S., and Harrison, M. (2010). Cetuximab and chemoradiation for rectal cancer-is the water getting muddy? Acta Oncol. (Madr.) 49, 278-286.

Hofheinz, R., Wenz, F. K., Post, S., Matzdorff, A., Laechelt, S., Hartmann, J. T., Müller, L., Link, H., Moehler, M. H., Kettner, E., Fritz, E., Hieber, U., Lindemann, $\mathrm{H}$. W., Grunewald, M., Kremers, S., Constantin, C., Hipp, M., Gencer, D., Burkholder, I., and Hochhaus, A. (2011). Capecitabine (Cape) versus 5-fluorouracil (5-FU)-based (neo)adjuvant chemoradiotherapy (CRT) for locally advanced rectal cancer (LARC): long-term results of a randomized, phase III trial. J. Clin. Oncol. 29, abstr. 3504.

Huang, S. M., and Harari, P. M. (2000). Modulation of radiation response after epidermal growth factor receptor blockade in squamous cell carcinomas: inhibition of damage repair, cell cycle kinetics, and tumor angiogenesis. Clin. Cancer Res. 6, 2166-2174.

Koeberle, D., Burkhard, R., von Moos, R., Winterhalder, R., Hess, V., Heitzmann, F., Ruhstaller, T., Terraciano, L., Neuweiler, J., Bieri, G., Rust, C., and Toepfer, M. (2008). Phase II study of capecitabine and oxaliplatin given prior to and concurrently with preoperative pelvic radiotherapy in patients with locally advanced rectal cancer. $\mathrm{Br}$. J. Cancer 98, 1204-1209.

Krook, J. E., Moertel, C. G., Gunderson, L. L., Wieand, H. S., Collins, R. T., Beart, R. W., Kubista, T. P., Poon, M. A., Meyers, W. C., Mailliard, J. A., Twito, D. I., Morton, R. F., Veeder, M. H., Witzig, T. E., Cha, S., and Vidyarthi, S. C. (1991). Effective surgical adjuvant therapy for high-risk rectal carcinoma. N. Engl. J. Med. 324, 709-715.

Lawrence, T. S., Blackstock, A. W., and McGinn, C. (2003). The mechanism of action of radiosensitization of conventional chemotherapeutic agents. Semin. Radiat. Oncol. $13,13-21$.
Lawrence, T. S., Davis, M. A., Tang, H.-Y., and Maybaum, J. (1996). Fluorodeoxyuridine-mediated cytotoxicity and radiosensitization require $\mathrm{S}$ phase progression. Int. J. Radiat. Oncol. Biol. Phys. 70, 273-280.

Roedel, C., Becker, H., Fietkau, R., Graeven, U., Hohenberger, W., Hothorn, T., Lang-Welzenbach, M., Liersch, T., Staib, L., Christiansen, H., Wittekind, C., Sauer, R., and German Rectal Cancer Study Group. (2011). Preoperative chemoradiotherapy and postoperative chemotherapy with 5-fluorouracil and oxaliplatin versus 5-fluorouracil alone in locally advanced rectal cancer: first results of the German CAO/ARO/AIO-04 randomized phase III trial. J. Clin. Oncol. 29, abstr. 3505.

Roh, M. S., Yothers, G. A., O'Connell, M. J., Beart, R. W., Pitot, H. C., Shields, A. F., Parda, D. S., Sharif, S., Allegra, C. J., Petrelli, N. J., Landry, J. C., Ryan, D. P., Arora, A., Evans, T. L., Soori, S., Chu, L., Landes, R. V., Mohiuddin, M., Lopa, S., and Wolmark, N. (2011). The impact of capecitabine and oxaliplatin in the preoperative multimodality treatment in patients with carcinoma of the rectum: NSABP-R04. J. Clin. Oncol. 29, abstr. 3503.

Sauer, R., Becker, H., Hohenberger, W., Rödel, C., Wittekind, C., Fietkau, R., Martus, P., Tschmelitsch, J., Hager, E., Hess, C. F., Karstens, J. H., Liersch, T., Schmidberger, H., Raab, R., and German Rectal Cancer Study Group. (2004). Preoperative versus postoperative chemoradiotherapy for rectal cancer. N. Engl. J. Med. 351, 1731-1740.

Shaalan Beg, M., Meyer, J., Balch, G. C., Xie, X.-J., and Singal, A. G. (2012). Pathologic complete response rates after neoadjuvant chemoradiation (CRT) for rectal cancer: do novel agents have a role? J. Clin. Oncol. 30, abstr. 597.

Willett, C. G., Boucher, Y., di Tomaso, E., Duda, D. G., Munn, L. L., Tong, R. T., Chung, D. C., Sahani, D. V., Kalva, S. P., Kozin, S. V., Mino, M., Cohen, K. S., Scadden, D. T., Hartford, A. C., Fischman, A. J., Clark, J. W., Ryan, D. P., Zhu, A. X., Blasekowsky, L. S., Chen, H. X., Shellito, P. C., Lauwers, G. Y., and Jain, R. K. (2004). Direct evidence that the VEGFspecific antibody bevacizumab has antivascular effects in human rectal cancer. Nat. Med. 10, 145-147. 
Willett, C. G., Duda, D. G., di Tomaso, E., Boucher, Y., Ancukiewicz, M., Sahani, D. V., Lahdenranta, J., Chung, D. C., Fischman, A. J., Lauwers, G. Y., Shellito, P., Czito, B. G., Wong, T. Z., Paulson, E., Poleski, M., Vujaskovic, Z., Bentley, R., Chen, H. X., Clark, J. W., and Jain, R. K. (2009). Efficacy, safety, and biomarkers of neoadjuvant bevacizumab, radiation therapy, and fluorouracil in rectal cancer: a multidisciplinary phase II study. J. Clin. Oncol. 27, 3020-3026.
Wolmark, N., Wieand, H. S., Hyams, D. M., Colangelo, L., Dimitrov, N. V., Romond, E. H., Wexler, M., Prager, D., Cruz, A. B. Jr., Gordon, P. H., Petrelli, N. J., Deutsch, M., Mamounas, E., Wickerham, D. L., Fisher, E. R., Rockette, H., and Fisher, B. (2000). Randomized trial of postoperative adjuvant chemotherapy with or without radiation for carcinoma of the rectum: National Surgical Adjuvant Breast and Bowel Project R-02. J. Natl. Cancer Inst. 92, 388-396.
Conflict of Interest Statement: The authors declare that the research was conducted in the absence of any commercial or financial relationships that could be construed as a potential conflict of interest.

Received: 02 February 2012; paper pending published: 06 March 2012; accepted: 27 April 2012; published online: 25 May 2012.

Citation: Engels B, Gevaert T, Sermeus $A$ and De Ridder M (2012) Current status of intensified neo-adjuvant systemic therapy in locally advanced rectal cancer. Front. Oncol. 2:47. doi: 10.3389/fonc.2012.00047

This article was submitted to Frontiers in Gastrointestinal Cancers, a specialty of Frontiers in Oncology.

Copyright (c) 2012 Engels, Gevaert, Sermeus and De Ridder. This is an open-access article distributed under the terms of the Creative Commons Attribution Non Commercial License, which permits non-commercial use, distribution, and reproduction in other forums, provided the original authors and source are credited. 